\title{
Acoustic levitation of planar objects using a longitudinal vibration mode
}

\author{
Yoshiki Hashimoto,* Yoshikazu Koike, ${ }^{* *}$ and Sadayuki Ueha** \\ *Kaijo Corporation, \\ 3-1-5, Sakaecho, Hamura, 205 Japan \\ **Precision and Intelligence Laboratory, Tokyo Institute of Technology, \\ 4259, Nagatsuda, Midori-ku, Yokohama, 227 Japan \\ (Received 21 October 1994)
}

Keywords: Acoustic levitation, Radiation pressure, Longitudinal vibration mode, Standing wave, Ultrasonic PACS number: 43. 25. Qp

\section{Introduction}

In conventional acoustic levitation experiments, weights of levitated objects were limited to no more than a few milligrams. This has prevented the acoustic levitation technique from being used in practical applications. So far, reflectors have been used to form a standing wave acoustic field within which objects were to be levitated on the node. The sizes of the objects, whose shapes are spherical or ellipsoidal rather than planar, are small compared with the wavelength used..$^{1-3)}$

If the surface of the object is planar, it can be used as a reflector itself and heavy objects with a such plan surface may be levitated. The authors experimentally found that such objects can be steadily levitated close to a piston-like radiation surface..$^{4-7)}$

In order to clarify the basic characteristics of levitation implemented by this method, the authors analyzed the properties of the levitation theoretically to compared with the experimental ones. According to the calculation, the distance of levitation should be directly proportional to the vibration amplitude and inversely proportional to the square root of the weight per unit area of the levitated object. The experiments proved that the measured data agreed with the theory in general and that planar objects of several kilograms to several dozen kilograms can be levitated regardless of the material. The maximum weight of levitated objects is roughly several thousand times greater than that in the former experiments.

\section{Theoretical analysis}

Consider the case where acoustic waves propagate from the radiating surface in the $a$ direction as shown in Fig. 1. In the following description, $a$ is a variable at the Lagrange coordinate and $x$ is a variable at the Euler coordinate. In Fig. 1, the radiation surface is assumed to be vibrating in harmonic motion as $\xi(0, t)=$ $\xi_{0} \cos (\omega t)$ and the bottom surface of the levitated object is assumed to be a rigid wall, that is $\xi(h, t)=0$. In addition to these assumptions, the dimensions of the surface and the bottom surface of the levitated object are assumed to be sufficiently large compared with the wavelength of the propagating acoustic waves.

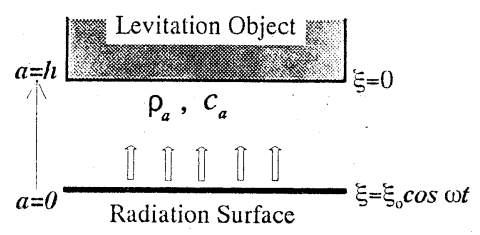

Fig. 1 Schema of acoustic levitation using longitudinal vibration mode.

The particle displacement distribution $\xi(a, t)$ at the Lagrange coordinate is expressed in Eq. (1) under the above conditions. This expression is approximated by the linear acoustic theory.

$$
\xi(a, t)=\xi_{0} \frac{\sin k(h-a)}{\sin k h} \cos \omega t
$$

In Eq. (1), $k$ denotes the wave number, $h$ is the distance between the radiation and the bottom surfaces and $\omega$ is the angular frequency.

Using the derivation of the density $\rho^{\prime}$ from the equation value, $\rho_{\mathrm{a}}$, the variation of the sound pressure $p^{\prime}$ is expressed as Eq. (2):

$$
p^{\prime}=c_{\mathrm{a}}{ }^{2} \rho^{\prime}
$$

In Eq. (2), $c_{\mathrm{a}}$ is the sound velocity in the medium.

By means of the relation between the Lagrange variable and the Euler variable, $x=a+\xi$, and the expansion of Eq. (2), Eq. (3) is obtained.

$$
\rho^{\prime}(x, t)=\rho^{\prime}(a+\xi, t)=\rho^{\prime}(a, t)+\frac{\partial \rho^{\prime}(a, t)}{\partial a} \xi
$$

In order to obtain the sound radiation pressure, Eq. (2) is taken the time average and the space average. The result is Eq. (4) by means of Eq. (3) and the integration by parts.

$$
\overline{p^{\prime}(a, t)}=-\int_{0}^{h} \overline{\rho^{\prime}(a, t) \frac{\partial \xi}{\partial a}} d a
$$

- represents the averageing operation. Using the linear approximation, $\rho^{\prime}(a, t)=-\rho_{\mathrm{a}}(\partial \xi / \partial a)$, and substitution of Eq. (1) into Eq. (4), Eq. (5) can be obtained. ${ }^{8,9)}$ 


$$
\overline{p^{\prime}}=\frac{1}{4} \rho_{\mathrm{a}} c_{\mathrm{a}}{ }^{2} \frac{\left(k \xi_{0}\right)^{2}}{\sin ^{2} k h}\left(1+\frac{\sin 2 k h}{2 k h}\right)
$$

In Eq. (5), if $h$ is sufficiently small compared with the wavelength of the acoustic waves $(k h \ll 1)$, $\sin k h$ approximately equals $k h$. After these approximations, we can obtain that the radiation pressure, which equals the levitated object weight per unit area. Then we obtain Eq. (6):

$$
w=\frac{1}{2} \rho_{\mathrm{a}} c_{\mathrm{a}}{ }^{2} \frac{\xi_{0}{ }^{2}}{h^{2}}
$$

where $w$ denotes the weight per unit area of the levitated object. Equation (6) states that levitation distance $h$ is inversely proportional to the square root of the weight per unit bottom area of the levitated object and is directly proportional to the vibration amplitude of the radiation plate. Equation (6) also states that the levitation distance does not depend on the vibration frequency and that any heavy object can be levitated if $h$ can be decreased infinitesimally without contact.

\section{Experiments}

In order to compare the theoretical analysis with experimental results, the following experiments were carried out.

3.1 Experimental set up and specifications of leviated objects

Figure 2 shows a basic configuration of the experimental setup. An object to be levitated was placed on the radiation surface of the radiator. The radiator is excited at a frequency of $19.22 \mathrm{kHz}$ with a conical horn which is driven by a sandwiched low frequency longitudinal transducer. The radiator is a rectangular parallelepiped and the dimensions of the radiation surface is $88.1 \times 124.0 \mathrm{~mm}$. The entire radiation surface was proved to be vibrating uniformly in phase and to

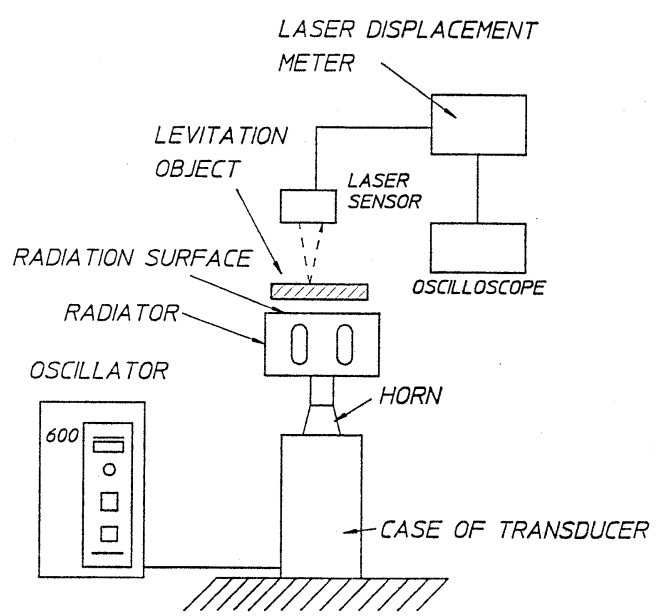

Fig. 2 The configuration of the experimental setup. have a minimum to maximum vibration ratio of 0.75 .

As planar levitation objects, bakelite plates and aluminum blocks were used in this series of experiments. Because the objects were to be levitated exploiting radiation pressure of acoustic waves, the bottom surface of the objects were to be flat and smaller than the radiation surface of the radiator. Table 1 lists the levitated objects.

The vibration amplitude of the radiation surface was measured with a high-frequency laser displacement meter and the value measured at the center was used as an experimental one. The levitation distance $h$ from the radiation surface was measured with a laser displacement meter. As the levitation of heavy metal blocks which were afloat about 200 micrometers or less could not confirm by eye, the verification that the object was completely off the radiation surface was carried out by measuring the electrical conductivity between the object and the radiation surface.

3.2 Levitation distance vs. weight of levitated object

Keeping the vibration amplitude of the radiation surface constant, each object listed in Table 1 was placed upon the radiation surface. Three peak-topeak vibration amplitudes: 10, 15, and 20 micrometers were employed. At each amplitude, objects having different unit-area weights were placed for levitation. And the measured levitation distance was plotted as a function of the weight of the object per unit area of bottom surface.

Figure 3 shows the results. The abscissa indicates the weights of levitated objects per unit bottom surface area, that is, the area that is directly supported by acoustic waves. As shown in the graph, it was proved

Table 1 The configurations of the levitation

\begin{tabular}{|c|c|c|c|}
\hline No. & $\underset{(\mathrm{kg})}{\text { Weight }}$ & $\begin{array}{l}\text { Weight per } \\
\text { unit area } \\
\left(\mathrm{kg} / \mathrm{m}^{2}\right)\end{array}$ & Material \& shape \\
\hline 1 & 0.0076 & 1.31 & \\
\hline 2 & 0.0118 & 2.03 & 90 \\
\hline 3 & 0.0237 & 4.07 & $\ln$ \\
\hline 4 & 0.0507 & 8.70 & \\
\hline 5 & 0.0793 & 13.60 & \multirow{2}{*}{$\begin{array}{l}\text { Bakelite plate thickness } \\
\qquad 1.0 \sim 15.2 \mathrm{~mm}\end{array}$} \\
\hline 6 & 0.1156 & 19.80 & \\
\hline 7 & 0.0139 & 2.83 & $\begin{array}{l}\text { Bakelite plate } \\
\qquad 70 \times 70 \times 2 \mathrm{~mm}\end{array}$ \\
\hline 8 & 0.1049 & 53.43 & Duralumin disk $\phi 50 \mathrm{~mm}$ \\
\hline
\end{tabular}
objects. 


\section{Y. HASHIMOTO et al.: ACOUSTIC LEVITATION OF PLANAR OBJECTS}

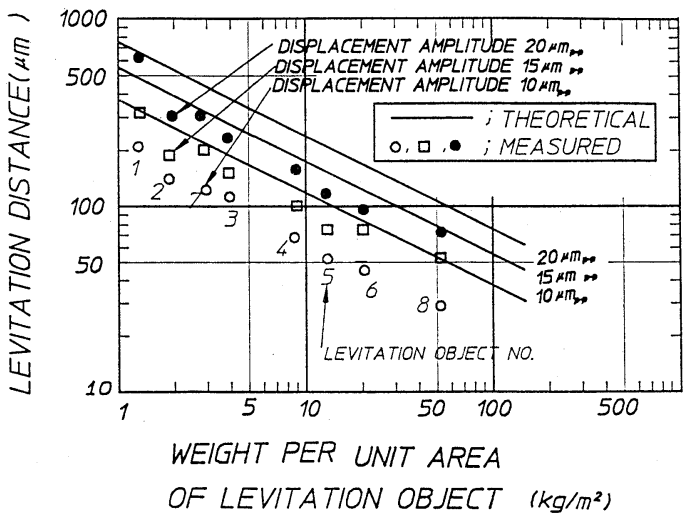

Fig. 3 Relationship between weight per unit area of a levitation object and levitation distance.

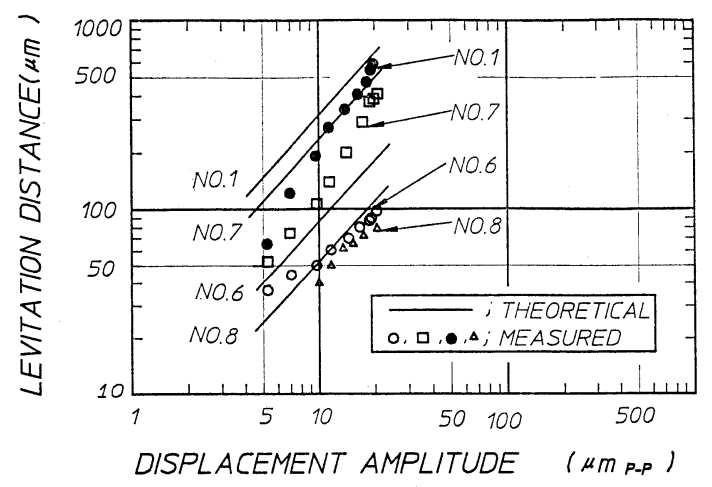

Fig. 4 Relationship between levitation distance and vibration amplitude at the center of the radiation surface.

that the distance of levitation is inversely proportional to the square root of the unit-area weight of the levitated object.

3.3 Levitation distances $v s$. vibration amplitudes

Changing the levitation object, the levitation distance $h$ was measured as a function of the vibration amplitude of the radiation surface. Figure 4 shows some examples of the result. It was proved from this figure that the distance of levitation is approximately proportional to the vibration amplitude.

\section{Discussion}

Solid lines in Figs. 3 and 4 show theoretical curves calculated in accordance with Eq. (6) to be compared with the experiment data. Constants used in theoretical calculation are: $c_{\mathrm{a}}=340 \mathrm{~m} / \mathrm{s}, \quad \rho_{\mathrm{a}}=1.0 \mathrm{~kg} / \mathrm{m}^{3}$, $g=9.8 \mathrm{~m} / \mathrm{s}^{2}$. The measured levitation distances are in fair agreement with theoretical calculations, proving that the Eq. (6) correctly expresses the levitation effect.
Possible causes of the difference between the measurements and expectations are:

(1) Piston-like motion of the radiation surface was imperfect.

(2) In Eq. (6), the bottom surfaces of the objects were assumed to be a rigid wall. Actually, however, some wave component escaped through the objects.

\section{Conclusions}

The authors found that sound waves can levitate weight several thousand times greater than previously proved. Experiment data showed fair agreements with theoretical one. Conclusions are:

1) The distance of levitation is inversely proportional to the square root of the levitated object weight per unit area of its bottom surface.

2) The distance of levitation is approximately proportional to the vibration amplitude of the radiation surface.

This levitation mechanism may possibly sustain and transfer objects contactlessly, without changing frequencies. If it does, industrial application of the technique in the future is very promising.

\section{Acknowledgments}

The authors would like to express their sincere thanks to Dr. M. Takeuchi, Tohoku University for his helpful suggestions and also to two persons belonging to Kaijo Corporation for valuable advises; Mr. Hisashi Fujita, the senior vice president and chief of the Industrial System Division, and Mr. Akio Fujie, the vice president and chief engineer.

\section{References}

1) Y. Aoyagi, H. Miura, and M. Kawamura, "Moving position of a small floating object by high intensity aerial sound wave," Proc. Spring Meet. Acoust. Soc. Jpn., 771-772 (1993).

2) T. Otsuka, M. Takei, and K. Seya, "Ultrasonic levitation for small particles by using stepped circular vibration plate," Proc. Spring Meet. Acoust. Soc. Jpn., 745-746 (1983).

3) H. Hatano, Y. Kanai, Y. Ikegami, T. Fujie, and K. Saito, "Ultrasonic levitation and positioning of samples," Jpn. J. Appl. Phys. 21 Suppl. 21-3, 202204 (1982).

4) Y. Hashimoto, Y. Koike, and S. Ueha, "Noncontact substance transportation using acoustic levitation," Proc. Autumn Meet. Acoust. Soc. Jpn., 901-902 (1993).

5) Y. Hashimoto, Y. Koike, and S. Ueha, "Noncontact substance transportation using acoustic levitation," IEICE Tech. Rep. US93-67 (1993).

6) Y. Hashimoto, Y. Koike, and S. Ueha, "Noncontact substance transportation using acoustic levitation II," Proc. Spring Meet. Acoust. Soc. Jpn., 1015-1017 (1994).

7) Y. Koike, Y. Hashimoto, and S. Ueha, "Noncon- 
tact substance transportation using acoustic levitation III," Proc. Spring Meet. Acoust. Soc. Jpn., 1067-1068 (1994).

8) J. Saneyoshi, Y. Kikuchi, and O. Nohmoto, Ultrasonic Technology Handbook (Nikkan Kogyoshinbunsha, Tokyo, 1991), p. 432.

9) L. D. Rozenberg, High-Intensity Ultrasonic Fields (Plenum Press, New York, 1971), pp. 85-97.

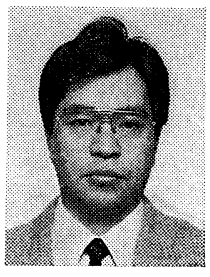

Yoshiki Hashimoto was born in Gunma prefecture on January 14, 1959. He received the B. Eng. degree in electric engineering, in 1981 from Ashikaga Institute of Technology, Ashikaga, Japan. Since 1981, he has been with Kaijo Co., Ltd., Tokyo, Japan, where he has been engaged in the development and application on high power ultrasonic systems. His research interests include ultrasonic cutter, ultrasonic cleaner and noncontact substance transportation systems. $\mathrm{He}$ is a member of The Acoustical Society of Japan.

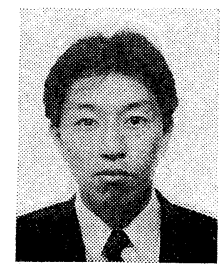

Yoshikazu Koike was born in Niigata prefecture on November 29, 1965. He received the B. Eng. and the M. Eng. degrees from Tokyo Institute of Technology, Tokyo, Japan, in 1989 and 1991 respectively. He has been a Research Associate of Precision and Intelligence Labora- tory, Tokyo Institute of Technology since 1993. His filed of research is application of ultrasonics and modal analysis. Mr. Koike is a member of The Acoustical Society of Japan.

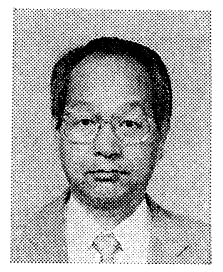

Sadayuki Ueha was born in Kyoto Prefecture on February 28, 1943. He received the B. Eng. degree in electric engineering from Nagoya Institute of Technology in 1965 and the M. Eng. degree in 1967 and the D. Eng. degree in 1970, both in electric engineering, from Tokyo Institute of Technology. He currently conducts research in high power ultrasonics, vibration measurement using physical optics, and acoustical imaging at the Tokyo Institute of Technology. Dr. Ueha is a vice president of the Acoustical Society of Japan and a member of The Japan Society of Applied Physics. The Institute of Electronics, Information, and Communication Engineers, and The Japan Society of Ultrasonics in Medicine. Currently, he is a member of the editorial board of The Journal "Ultrasonics." He received The Best Paper Awards from The Japan Society of Applied Physics in 1975 and from The Acoustical Society of Japan in 1980, respectively. 